\title{
Chronic Craniofacial Pain from Entrapment of the Greater Occipital Nerve Misdiagnosed as Chronic Migraine, Idiopathic Odontalgia, and Atypical Facial Pain
}

\author{
Chang-ik Lee ${ }^{1}$, Byung-chul Son ${ }^{1,2}$ \\ ${ }^{1}$ Department of Neurosurgery, Seoul St. Mary's Hospital, College of Medicine, The Catholic University of Korea, Seoul, \\ ${ }^{2}$ Catholic Neuroscience Institute, College of Medicine, The Catholic University of Korea, Seoul, Republic of Korea
}

Corresponding author: Byung-chul Son

Department of Neurosurgery,

Seoul St. Mary's Hospital,

Catholic Neuroscience Institute, College of Medicine, The Catholic University of Korea, 222

Banpo-daero, Seocho-gu, Seoul 06591, Republic of Korea

Tel: +82-2-2258-6122

Fax: +82-2-594-4248

E-mail: sbc@catholic.ac.kr

Received: September 17, 2019 Revised: September 18, 2019 Accepted: September 19, 2019
Although the cause of occipital neuralgia is mostly unknown, entrapment of the greater occipital nerve (GON) at its piercing point of the tendinous aponeurotic attachment of the trapezius at the superior nuchal line has been reported to be the most common cause. We report an occurrence of unilateral facial pain associated with continuous aching and stabbing headache in the frontotemporal and occipital regions in a patient whose occipital neuralgia has lasted for years. These symptoms were completely different from those of typical occipital neuralgia, making diagnosis very difficult. A 52-year-old male patient with a 6-year history of intermittent stabbing pain in his right suboccipital area presented with an unremitting continuous pain in his right frontotemporal and malar areas that lasted 5 months. The aching and stabling pain in his frontotemporal and malar areas developed suddenly 5 months prior to presentation. Although he was treated after visiting neurology, dentistry, opthalmology, ENT, and pain clinic, the pain was not controlled. It spread to right periorbital and right occipital areas. At 3 months following decompression of the right GON, he reported no more pain and was able to stop the medication. Until 12 months after the operation, his craniofacial pain did not recur.

Key Words: Decompression; Facial pain; Migraine disorders; Pain; Trigeminal neuralgia

\section{INTRODUCTION}

Occipital neuralgia is characterized by paroxysmal shooting or stabling pain in the posterior part of the scalp with distributions of the greater and lesser occipital nerves (GON, LON). It often accompanied by dysesthesia and tenderness ${ }^{7}$. Diagnosis and treatment of occipital neuralgia are usually not difficult if we are aware of its characteristic symptoms and signs. The etiology of occipital neuralgia is mostly unknown ${ }^{18)}$. However, entrapment of the GON at its piercing point of the tendinous aponeurotic attachment of the trapezius at the superior nuchal line is known to be the most common cause $6,12,15,18)$

Occipital neuralgia is not a sole manifestation of compression/ entrapment of the GON. We experienced diverse presentations of craniofacial pain, in addition to paroxysmal stabbing pain of occipital neuralgia from compression/entrapment of the GON during the last 3 years ${ }^{8,9,11,14,15)}$. From our positive experience of more than 100 cases of GON decompression, we have realized that constant aching pain, in addition to paroxysmal stabbing pain, are also common in GON entrapment. Furthermore, more than one-third of patients with GON entrapment have presented with continuous aching pain in the face that is well known to be the distribution of the trigeminal nerve $^{14)}$

Many patients with craniofacial pain caused by chronic entrapment of the GON have been treated under multiple diagnoses such as migraine, tension headache, atypical facial pain, odontalgia, periorbital pain, and pain from temporomandibular joint disorders. We have demonstrated several cases regarding hemifacial, trigeminal referred pain from chronic GON entrapment $^{8,9,11,14,15)}$. Without knowledge and information on trigeminal referred facial pain from GON entrapment, many patients are still suffering from long-term ineffective treatment. To enhance understanding of chronic GON entrapment, we report a patient with chronic craniofacial pain treated as multiple diagnoses of migraine, tension headache, odontalgia, and atypical facial pain. His chronic, unremitting pain was successfully relieved with decompression of the GON.

\section{CASE REPORT}

A 52-year-old, right-handed male patient with a 6-year history of intermittent stabbing pain in his right occipital area presented with an unremitting continuous pain in his right frontotem- 

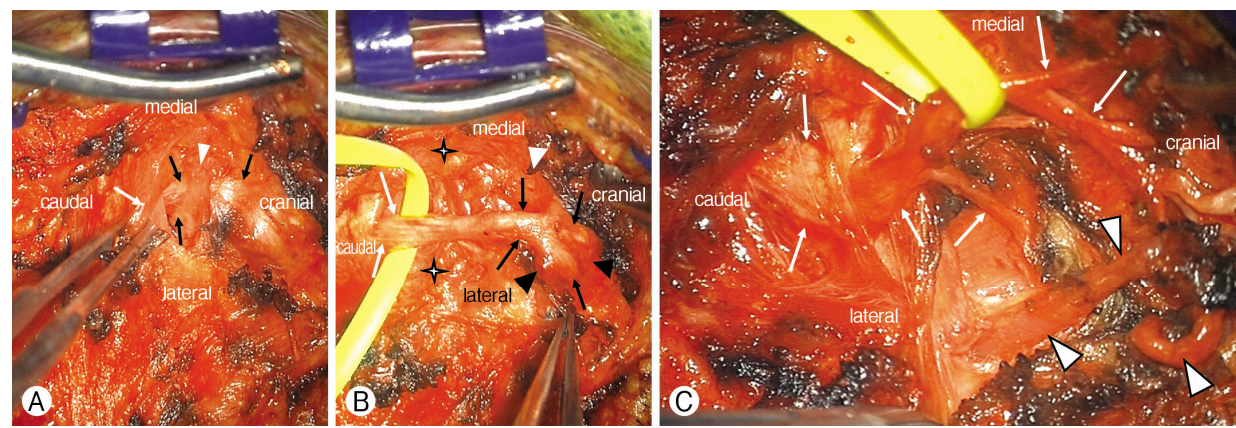

Fig. 2. Intraoperative findings during decompression of the greater occipital nerve (GON). (A) Retraction of the tendinous aponeurotic edge of the trapezius (white arrow) showing an indented GON (black arrows) and constricting fascial band (white arrowhead). (B) Decompression of tendinous aponeurotic edge (white stars) revealing proximal course (black arrows) of the GON within the semispinalis capitis muscle. The distal GON (black arrowheads) was severely adherent to the underlying occipital artery (black arrowheads). Note the remained fascial band (white arrowhead) constricting the GON. (C) An intraoperative photograph showing fully dissected course of the GON (arrows) from trapezial ring and the occipital artery (arrowheads).

poral and malar areas (Fig. 1A, B). His pain in frontotemporal and malar areas developed suddenly 5 months ago without any precipitating cause. It had continued since it first broke out. The nature of pain was described as aching and stabbing. It lasted all day. It was more severe at night. Its intensity was rated as 4 to 5 out of 10 in numerical rating scale (NRS-11) during daytime and 6 to 7 out of 10 in NRS-11 at night. One month before presentation, sensation of swelling and pressure rising of the right periorbital area developed insidiously (Fig. $1 \mathrm{~B})$. In addition, aching and stabbing pain in the right-side occipital area aggravated. Especially, it was so painful that he could not use the pillow at night. Two weeks ago, pressure sensation in the right periorbital area was worsened with lacrimation and conjunctival injection.
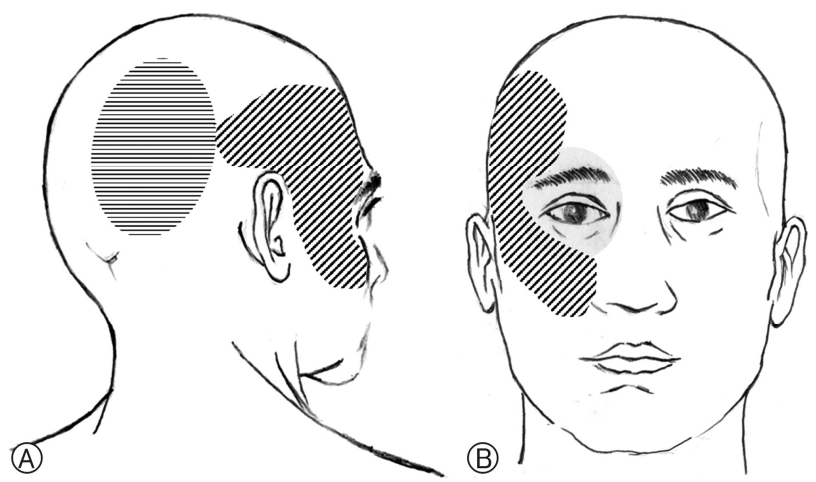

Fig. 1. Diagrams demonstrating the distribution of craniofacial pain. (A) Horizontally hatched area over the right occipital area indicates the distribution of aching and stabbing pain. Obliquely hatched area over the right frontotemporal and malar regions indicates the distribution of aching and stabbing pain. (B) Dark area over the right periorbital region indicates distribution of pain with swelling and pressure sensation. III-defined sensation of congestion and ipsilateral lacrimation were associated with each pain attack.
He was treated at the dentist's office because of a sore malar area without a particular cause. He was diagnosed with idiopathic odontalgia and treated with medication. Then, he was diagnosed as migraine at a nearby hospital, he took nonsteroidal anti-inflammatory drugs (Ultracet ${ }^{\mathbb{Q}}$ ) and headache pills (propranolol, triptans, and cafergot). However, these medications had no significant effect on the pain. The pain was so bad that he could not sleep at night. He said he was treated for eye pain by an ophthalmologist, although there was nothing wrong with the eye. The otolaryngology doctor also said there was no problem. Other neurologist had diagnosed and treated him for a tension headache and atypical facial pain. Although he was treated in so many hospitals, his headache and facial pain gradually got worse. Thus, he visited our outpatient clinic.

In the past history, 6 years ago, he suffered from pain in the right occipital area for a year. Tingling and stabbing pain persisted throughout the day. In several cases, he had to visit the emergency room to get an analgesic injection. That year, he had pain for more than 15 days a month. Although he was treated in several hospitals, treatment did not work very well. After a year or so, the severe occipital pain disappeared on its own. Since then, there had been pain in the right occipital area 2 to 3 times a year, each time lasting for 1 to 2 weeks. The pain was not as bad as before. It even disappeared on its own after a while. He had no particular medical conditions such as hypertension and diabetes in past history. He denied any trauma to the neck or cervical sprain. He had general office work, not physical work.

In physical examination, there was no objective sensory impairment on the face or head. There was no abnormality in cranial nerve examinations either. A slight tenderness was found around the right nuchal line. A mild allodynia to light touch was observed in the occipital region. There was no res- triction on the movement of the neck. The pain had not worsened to affect neck movement. Unusually, when he lied back, his craniofacial pain got worse. 
Thus, he slept only on his side. There were no symptoms or signs of trigeminal autonomic cephalalgia, including photophobia, nausea, vomiting, or conjunctival injection. However, the patient had lacrimation and subjective feeling of malar swelling during attack of right facial and frontal pain. No specific dental cause or paranasal sinusitis was identified. No pathologic lesion was found to explain his left facial and occipital pain in enhanced magnetic resonance imaging of the brain and cervical spine conducted previously.

His chronic facial and occipital pain was thought to be a pain associated with the GON. Thus, $2 \mathrm{~mL}$ of lidocaine was injected for occipital nerve block (ONB). The right occipital pain improved for an $\mathrm{hr}$ following the ONB. However, the pain in the temporal and malar area did not improve. Considering chronicity and referred nature of his frontal and malar pain associated with preexisting occipital neuralgia, exploration of the right GON was proposed.

A $4 \mathrm{~cm}$-sized linear incision was made obliquely along the course of right GON. The incision was then developed over the trapezius fascia. The proximal course of the right GON was identified between the trapezius and semispinalis capitis muscles (Fig. 2). After proximal dissection of semispinalis capitis and trapezius muscles from the GON, it was then carried distally, releasing the GON within the trapezial canal (the site where the GON penetrated through the trapezial aponeurotic attachment to the occiput at the superior nuchal line). The GON was found to be severely entrapped by the aponeurotic arch of the trapezius (Fig. 2). There was an indentation in the GON for chronic entrapment. The occipital artery was severely adherent to the GON at the trapezial canal. It was dissected free from the GON. The tightly adhered segment of the occipital artery was cut. After complete decompression of the GON, overlying wound was closed in two layers.

The morning after the decompression, the periorbital pain disappeared. Within seven days, chronic aching pain in his frontotemporal and malar area was considerably relieved. Up to a month after surgery, there was discomfort in the occipital vertex. It was a bit severe at night. Numbness in the occipital vertex was controlled with $200 \mathrm{mg}$ /day of gabapentin. At 3 months, he stopped taking medication because he felt no discomfort at all. No pain in the frontotemporal, malar, or periorbital area was noted. Subjective satisfaction of the patient was very high. He reported good condition without craniofacial pain in six-month and 12-month follow-up.

\section{DISCUSSION}

\section{Greater Occipital Nerve and Its Entrapment}

The GON refers the medial branch of the dorsal ramus of the second cervical nerve ${ }^{16,17)}$. It may also have fibers derived from the dorsal ramus of $\mathrm{C} 3$. The GON ascends through the semispinalis and then runs rostrolaterally before emerging in- to the scalp by piercing the aponeurotic fibrous attachment of the trapezius to the superior nuchal line $e^{4,6,11,12,16,17)}$. This aperture (trapezial canal) is a common site of entrapment of the $\mathrm{GON}^{4,12,15,16)}$. At this point, it travels with the occipital artery to supply the integument of the scalp as far anterior as the vertex ${ }^{17)}$. In this case, chronic pain in the right-sided hemiface; malar and periorbital pain in addition to the frontotemporal areas were caused by entrapment of the right GON at the trapezial canal. In addition to entrapment within the tendinous aponeurotic attachment of the trapezius, the GON was found to be densely adhered to the occipital artery. The occipital artery, accompanied by the GON, turns upwards to pierce the investing layers of the deep cervical fascia connecting to the cranial attachment of trapezius and sternocleidomastoideus, and ascends tortuously in the dense superficial fascia of the scalp where it divides into many branches ${ }^{16)}$. Compression of the GON by the occipital artery within the trapezial canal was reported to be the cause of occipital neuralgia in 2011 and $2017^{5,14)}$. Although entrapment of the GON by the fascial ring was a major intraoperative pathological finding, dense arterial adhesion could be non-negligible cause of GON entrapment. We think that both fascial entrapment and arterial adhesion should be addressed to decompress the GON completely.

Clinical significance of the GON entrapment at and around the trapezial canal has been stressed repetitively during the last two decades. It is a well known etiology of occipital neuralgia $^{8,11,14,17,18)}$. In addition, entrapment of the GON was reported to be associated with cervicogenic headache, migraine headache ${ }^{12)}$, chronic headache ${ }^{6}$, and referred trigeminal pain from occipital neuralgia ${ }^{8,11,14)}$. Even hemifacial sensory change in addition to referred trigeminal pain caused by entrapment of the GON has been reported ${ }^{14)}$.

\section{Differential Diagnosis of Chronic GON Entrapment}

Diagnosis of chronic entrapment of the GON was typically delayed in the current case. The reason for delayed diagnosis is the presence of pain in the malar (V2) and periorbital (V1) in addition to frontotemporal headache. Due to the unexplained, chronic aching and stabbing pain in his malar area, he was evaluated and treated by a dentist and an otolaryngology doctor. He was also evaluated by an ophthalmologist for periorbital pain. He was mainly treated by neurologists for frontotemporal headache for several months.

His headache in the frontotemporal area was treated under the diagnosis of chronic migraine and tension headache. Indeed, this headache occurred more than 15 days/month for more than 3 months and last more than 4 hr a day. Furthermore, it was unilateral and its severity was moderate to severe. However, it did not show the pulsating quality of typical migraine. Routine physical activity did not affect the pain either. There was no nausea, vomiting, or photophobia. Thus, it is difficult 
to diagnose this headache as a migraine. When it comes to tension headache, there is mild pericranial tenderness over the occipital area on palpation. However, the headache was typically unilateral. In addition, hemifacial pain in periorbital and malar area was difficult to explain. Medication-use headache or new daily persistent headache can be ruled out by typical history of the patient.

Hemifacial pain in his periorbital and malar area was different from that of idiopathic trigeminal neuralgia. His facial pain was continuous rather than paroxysmal. It was not triggered by innocuous stimuli. Although he described it as aching and stabbing, it was not electric shock-like in the nature of pain. In addition, there was no discernable vascular structure abutting the right trigeminal nerve in magnetic resonance imaging examination. Thus, the hemifacial pain was diagnosed as persistent idiopathic facial pain (formerly called atypical facial pain). Organic causes of eye, nose, teeth, or temporomandibular disease were excluded.

Indeed, the right occipital pain that the patient experienced 6 years ago was a typical occipital neuralgia. However, he did not receive an exact explanation from doctors about the diagnosis. He only received symptomatic treatment. The chronic prognosis of occipital neuralgia is not well known. Considering that entrapment of the GON at the trapezial ring is the most common cause of occipital neuralgia, occurrence of hemifacial pain as well as frontotemporal pain in this patient can be the long-term consequence of chronic GON entrapment. This prediction can be judged to be credible as the patient's symptoms have improved through decompression of the GON. We have experienced several cases that hemifacial pain, in addition to the occipital neuralgia, is improved by decompression of the $\mathrm{GON}^{8,9,11,14,15)}$. Although it has already been mentioned that the pain of occipital neuralgia may be referred to the fronto-orbital area through trigeminocervical interneuronal connections in the trigeminal spinal nuclei ${ }^{1)}$, referred facial pain in addition to occipital neuralgia has not been accurately reported ${ }^{8,9,11,14,15)}$.

\section{Referred Trigeminal Pain from Chronic GON Entrapment}

Referred pain in the facial trigeminal area from occipital neuralgia can occur even in V2 distribution in addition to fronto-orbital (V1) area ${ }^{11)}$. Although it is difficult to draw a comprehensive conclusion from a single case report ${ }^{11)}$, it is possible that these examples have been under-reported. Therefore, they are not well understood by health care physicians. If referred facial pain caused by chronic GON appears more common than we thought and if the symptoms of GON entrapment appears to be continuous aching pain rather than paroxysmal, shooting, stabbing pain which is typical of occipital neuralgia, then facial pain of GON entrapment is very ambi- guous and difficult to diagnose. In order to inform this fact, we decided to report this instance.

Findings of current case provide clinical affirmation of the existence of trigeminal/cervical convergence and hypersensitivity. Patients with primary headaches often report pain involving not only the frontal head innervated by the trigeminal nerve but also the occipital region innervated by the $\mathrm{GON}^{13)}$. Stimulation of structures in the neck innervated by upper cervical roots such as infratentorial dural mater, vessels, and tumors of the posterior fossa in humans can lead to pain in the frontal head ${ }^{1,13}$. These findings are related to sensitization and hyperactivity of trigeminocervical complex (TCC). TCC is a population of neurons in the $\mathrm{C} 2$ dorsal horn that receive convergent input from facial skin corresponding to the trigeminal nerve and cervical structures innervated by high cervical C1-3 roots ${ }^{1,2,13)}$. Convergence of nociceptive afferents and sensitization of TCC neurons have clinical correlates, including hypersensitivity and the spread and referral of pain frequently seen in patients with primary headaches such as migraines ${ }^{1,2,13)}$. Chronic noxious afferent input from chronic entrapment of the GON may lead to central sensitization of the TCC and result in referred hemifacial trigeminal pain ${ }^{1,2,13,14)}$.

\section{Entrapment of GON Associated with Activation and Sensitization of Extracranial Nociceptor}

Tenderness and allodynia in the occipital area were associated with frontotemporal headache in the patient of the present case. These symptoms and signs were successfully treated with decompression of the GON. Occipital, pericranial tenderness caused by chronic GON entrapment might be associated with activation of extracranial nociceptors ${ }^{3)}$. Activation of extracranial nerves or tissues that they innervate (e.g., calvarial bone and periosteum, pericranial muscles, and fascia surrounding nerves) is related to migraine attack $^{3)}$. Anatomical connections between intracranial meningeal and extracranial nociceptive fibers within periosteum and pericranial muscles have been found ${ }^{10)}$. These fibers constitute a network of sensory (peripherin-positive) and pain (calcitonin gene related peptide and transient receptor potential cation channel subfamily $\mathrm{V}$ member 1-positive) fibers that traverse calvarial bones along suture lines in young mice ${ }^{3,10)}$. Therefore, pain signals originating in the periosteum, pericranial muscles, fascia surrounding nerves, and nerves themselves can reach trigeminal pain-sensitive sensory fibers, causing activation of trigeminovascular nociceptors. In addition, trigeminal sensory fibers are capable of conducting action potentials in both orthodromic and antidromic directions. Chronic nociceptive input from entrapment of extracranial nerves that supply the head (GON and LON, posterior auricular, auriculotemporal and zygomaticotemporal, supraorbital nerves) can sensitize the TCC in C2 spinal cord. This sensitization can evoke a headache with features similar to those of migraine in frontotemporal area. 


\section{CONCLUSION}

A continuous aching and stabbing pain in frontotemporal, malar, and occipital regions in a patient with past history of occipital neuralgia was treated with decompression of the GON. The diagnosis was delayed despite multiple medical examinations. Referred facial pain from chronic entrapment of the GON should be kept in mind in patients with unexplained, unremitting head and neck pain.

\section{CONFLICTS OF INTEREST}

No potential conflict of interest relevant to this article was reported.

\section{REFERENCES}

1. Bartsch T, Goadsby PJ: Stimulation of the greater occipital nerve induces increased central excitability of dural afferent input. Brain 125:1496-1509, 2002

2. Bartsch T, Goadsby PJ: The trigeminocervical complex and migraine: current concepts and synthesis. Curr Pain Headache Rep 7:371-376, 2003

3. Burstein R, Blake P, Schain A, Perry C: Extracranial origin of headache. Curr Opin Neurol 30:263-271, 2017

4. Cesmebasi A, Muhleman MA, Hulsberg P, Gielecki J, Matusz P, Tubbs RS, et al.: Occipital neuralgia: anatomic considerations. Clin Anat 28:101-108, 2015

5. Cornely C, Fischer M, Ingianni G, Isenmann S: Greater occipital nerve neuralgia caused by pathological arterial contact: treatment by surgical decompression. Headache 51:609-612, 2011

6. Ducic I, Moriarty M, Al-Attar A: Anatomical variations of the occipital nerves: implications for the treatment of chronic headaches. Plast Reconstr Surg 123:859-863, 2009

7. Headache Classification Committee of the International Headache Society: Headache Classification Committee of the Inter- national Headache Society (IHS) The International classification of headache disorders, 3rd edition. Cephalalgia 38:1-211, 2018

8. Ko HC, Choi JG, Son BC: Hemifacial trigeminal pain referred from occipital neuralgia due to compression of the greater occipital nerve by the occipital artery. J Neurol Surg A Cent Eur Neurosurg 79:442-446, 2018

9. Ko HC, Son BC: Chronic unilateral temporo-occipital headache attributed to unilateral C3 radiculopathy. Asian J Neurosurg 13:1229-1232, 2018

10. Kosaras B, Jakubowski M, Kainz V, Burstein R: Sensory innervation of the calvarial bones of the mouse. J Comp Neurol 515: 331-348, 2009

11. Lee CI, Son BC: V2 trigeminal referred pain from chronic occipital neuralgia caused by entrapment of the greater occipital nerve. Indian J Neurosurg 8:76-80, 2019

12. Mosser SW, Guyuron B, Janis JE, Rohrich RJ: The anatomy of the greater occipital nerve: Implications for the etiology of migraine headaches. Plast Reconstr Surg 113:693-697; discussion 698-700, 2004

13. Piovesan EJ, Kowacs PA, Tatsui CE, Lange MC, Ribas LC, Werneck LC: Referred pain after painful stimulation of the greater occipital nerve in humans: evidence of convergence of cervical afferences on trigeminal nuclei. Cephalalgia 21:107-109, 2001

14. Son BC, Choi JG: Hemifacial pain and hemisensory disturbance referred from occipital neuralgia caused by pathological vascular contact of the greater occipital nerve. Case Rep Neurol Med 2017:3827369, 2017

15. Son BC, Kim DR, Lee SW: Intractable occipital neuralgia caused by an entrapment in the semispinalis capitis. J Korean Neurosurg Soc 54:268-271, 2013

16. Standring S: Gray's Anatomy: The anatomical basis of clinical practice, ed 40th. London, UK: Churchill Livingstone Elsevier, 2008

17. Tubbs RS, Salter EG, Wellons JC, Blount JP, Oakes WJ: Landmarks for the identification of the cutaneous nerves of the occiput and nuchal regions. Clin Anat 20:235-238, 2007

18. Vanelderen P, Lataster A, Levy R, Mekhail N, van Kleef M, Van Zundert J: 8. Occipital neuralgia. Pain Pract 10:137-144, 2010 